\title{
Double active region index-guided semiconductor laser
}

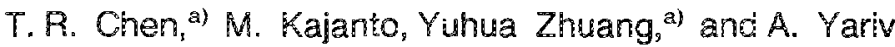 \\ California Institute of Technology, Pasadena, California 91125
}

(Received 10 August 1988; accepted for publication 31 October 1988)

\begin{abstract}
A buried crescent $\mathrm{MGaAsP} / \mathrm{InP}$ laser with a double active layer was fabricated. The laser showed very high characteristic temperature $T_{0}$ and highly ronlinear light versus current characteristics. A theoretical model using a rate equation approach showed good agreement with the experimental results.
\end{abstract}

There has been continuous interest in making InGaAs?/InPl lasers with a high characteristic temperature $T_{0}{ }^{1-5}$ A promising approach seems to be the double carrier confinement (DCC) structure. ${ }^{4,5}$ In this structure a second "active" layer is grown to collect the carriers which leak out of the first active layer and to reuse them for radiative recombination. $T_{0}$ as high as $180 \mathrm{~K}$ has been repored. ${ }^{4,5}$ However, the idea has only been demonstrated in broad-area (or wide stripe) lasers. It is thus of great interest to see if the idea is applicable to the commoniy used laser structures, e.g., buried heterostructure (BH) or buried crescent (BC) 12 sers.

In this letter, we report on the first attempt to build a real index-guided laser with two active layers. In addition to the increase of characteristic temperature, we found highly nonlinear behavior in the light versus current $(L-I)$ characteristics of the DCC laser. A theoretical model shows good agreement with the experimental data.

Buried crescent (BC) structures were used for our study. Lasers were made on both an $n$-InP substrate and $p$ InP substrate. A schematic structure of the laser is shown in Figs. 1(a) and (b). A scamning electron microscope (SEM) picture of the double active layer is shown in Fig. 1(c). The fabrication processes of the laser involve two-step liquid phase epitaxy (LPE) growth which has been described in great detail in the literature. ${ }^{6}$ The only diference is the growth of two active layers by "three-melt technique." In the whole second growth cycle only one

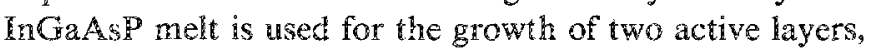
which ensures the exact overlap of the transition gain profile of the two kayers.

The threshold currents of the $\mathrm{HC}$ DCC lasers on $n$ - $\mathrm{nP}$ substrate are in the range of $20-45 \mathrm{~mA}$ for a cavity length of $\sim 250 \mu \mathrm{m}$. Lasers made on the same wafer with a single active layer had a threshold current of $\sim 10-15 \mathrm{~mA}$. For the $\mathrm{BC}$ DCC laser on the $p$ - In $\mathrm{P}$ substrate, the threshold currents are $40-60 \mathrm{~mA}$, while threshold currents of lasers made from the same solution with single active layers are typically i 5 $20 \mathrm{~mA}$. The emitting wavelength of the $\mathrm{BC}$ DCC laser is $\sim 1.3 \mu \mathrm{m}$. For the wafers we processed, the thicknesss of the active layer is $\sim 0.15-0.25 \mu \mathrm{m}$, the separation between the two active layers is $0.05-0.35 \mu \mathrm{m}$, and the width of the active layer is $2-2.5 \mu \mathrm{m}$.

The main feature of the light output versus current characteristics $(L-I$ curve $)$ is the high nonlirearity. Figure 2 compares the $\mathrm{cw} L-I$ curves of a typical $\mathrm{BC}$ DCC laser and a

"On leave from University of Electronics Science and Technology, China. single active layer $B C$ laser. The $L-I$ curve of the conventional BC laser shows good linearity while the $B C D C C$ laser displays optical switching behavior. An optical power jump of $2-3 \mathrm{~mW}$ at the threshold for BC DCC lasers was observed. The external quantum efficiency in this region is

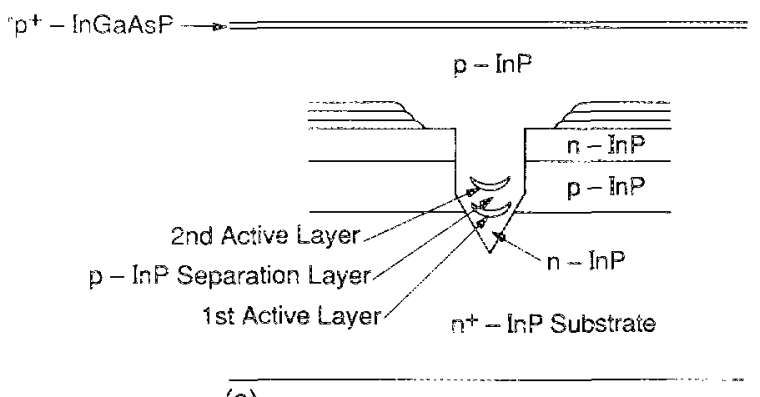

(a)

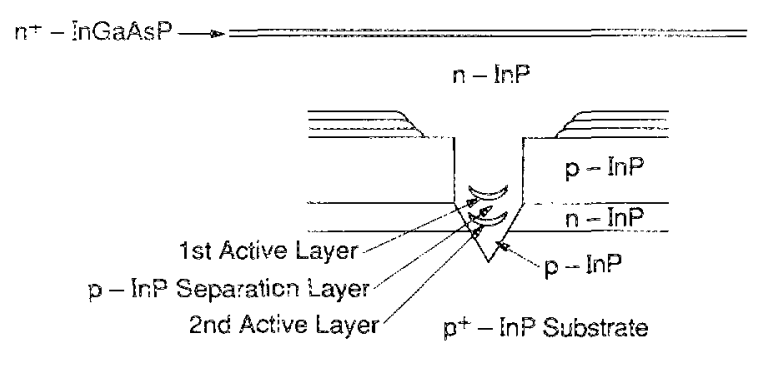

$\overline{(b)}$

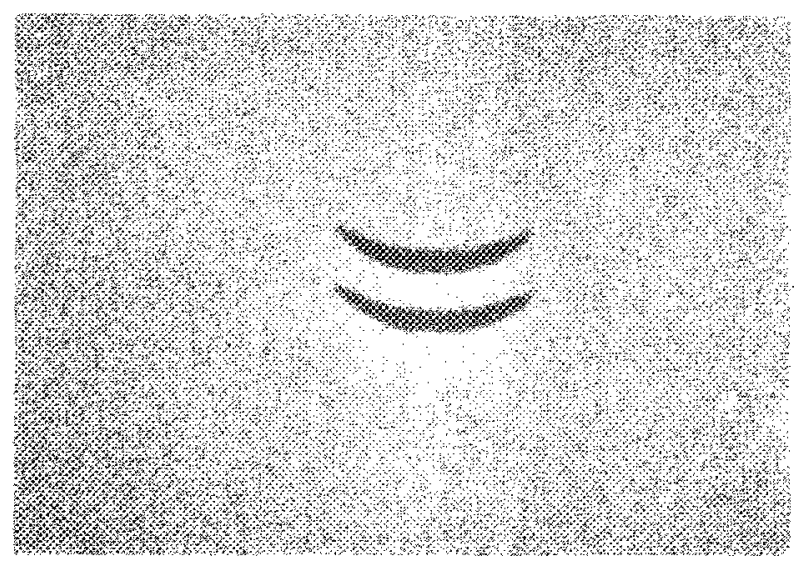

(c)

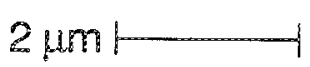

FIG. 1. (a) Schematic structure of BC DCC laser on $n$-In substrate. (b) Schematic structure of $3 C$ DCC laser on $p$-InP substrate. (c) SEM picture of the double active region. 


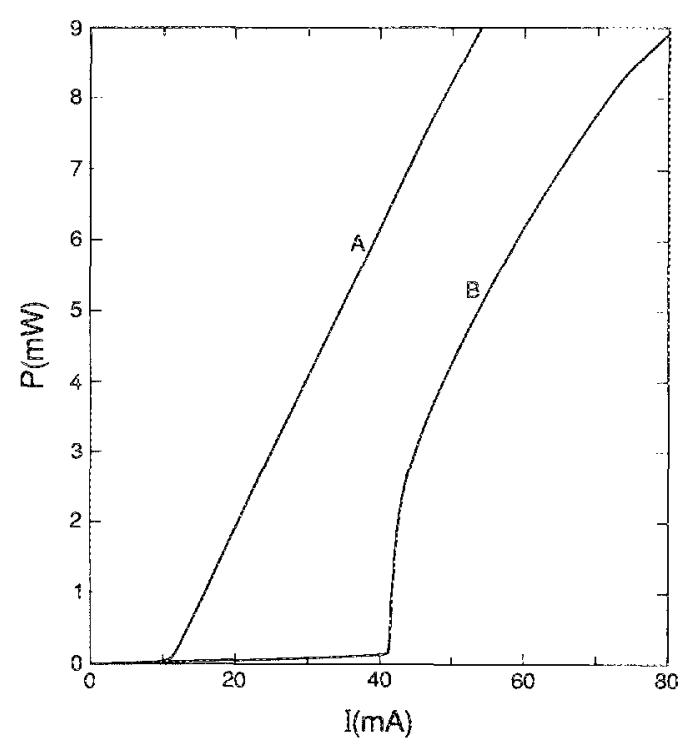

FIG. 2. Light vs current curves of BC lasers. (a) BC laser with single active layer. (b) BC DCC laser.

much higher than that of the single active layer $B C$ laser, and it can be larger than $100 \%$ per facet. After the initial swich, there is a region in which the $L-I$ curve of the $B C D C C$ laser has comparable slope as that of the single active layer $B C$ laser. Then the optical power gradually saturates. The power of the BC DCC laser saturates much faster than the conventional $\mathrm{BC}$ laser.

The temperature dependence of the threshold current of the $\mathrm{BC}$ laser was examined. Assuming $I_{\text {it }} \propto e^{T / T_{0}}$, the characteristic temperature $T_{0}$ for our single active layer $B C$ lasers is typically $60 \mathrm{~K}$. For the $\mathrm{BC}$ DCC laser on $p$-InP substrate, measured $T_{0}$ ranges from 120 to $250 \mathrm{~K}$ (Fig. 3 ), depending on the laser parameters (e.g., the thickness of the active layers and the separation between the two active layers). The measured $T_{0}$ for the BC DCC laser on the $n$ - InP substrate is somewhat lower, but is still $\sim 30 \mathrm{~K}$ higher than that of the single active layer $B C$ laser. The possible reason is that the separation between the two active layers for the wafer we

xessed is too small. Another possibility is the shift of the ction due to $\mathrm{Zn}$ diffusion during the growth. ${ }^{5}$

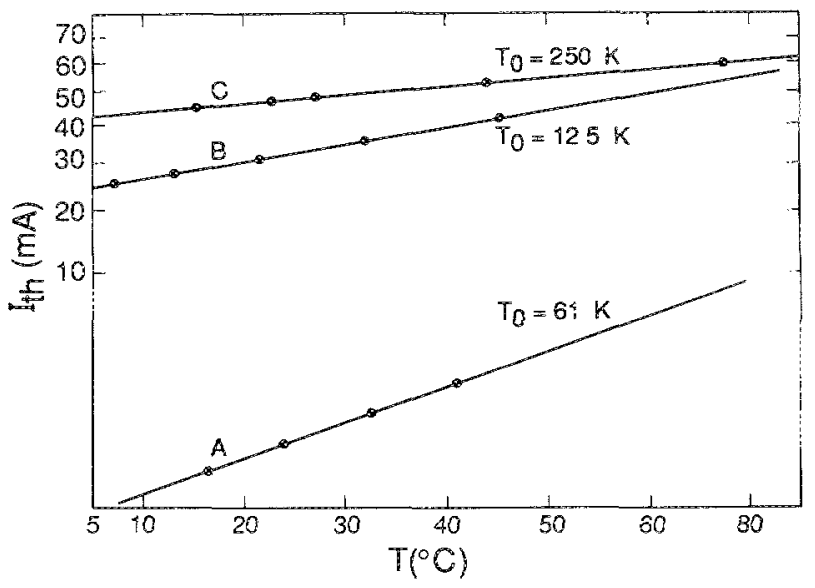

FIG. 3. $I_{1 \text { t: }}$ of RC lasers as a function of temperature, showing different $T_{0}$ values. (a) BC laser with single active layer. (b), (c) BC DCC laser on $p$ inP substrate.
For better understanding the BC DCC laser, an anaiysis using rate equations has been carried out. The equations which govern the carrier density $N_{i}$ in the th active layer and photon density $P$ in the lasing mode are

$$
\begin{aligned}
\frac{d N_{1}}{d t}= & \frac{J_{1}}{e d_{1}}-B\left(N_{1}+N_{A 1}\right) N_{1} \\
& -A\left(N_{1}-N_{\mathrm{tr}}\right) P-R_{\mathrm{Auger}, 1}, \\
\frac{d N_{2}}{d t}= & \frac{J_{2}}{e d_{2}}-B\left(N_{2}+N_{A 2}\right) N_{2} \\
& -A\left(N_{2}-N_{\mathrm{tr}}\right) P-R_{\mathrm{Auger}, 2}, \\
\frac{d P}{d t}= & A\left[\Gamma_{t}\left(N_{1}-N_{\mathrm{tr}}\right)+\Gamma_{2}\left(N_{2}-N_{\mathrm{tr}}\right)\right] P-\frac{P}{\tau_{p}} \\
& +\beta B\left[\Gamma_{1} N_{\mathrm{t}}\left(N_{1}+N_{A 1}\right)+\Gamma_{2} N_{2}\left(N_{2}+N_{A 2}\right)\right], \\
J_{\text {I.eak }, 1}= & J_{2}+J_{\text {leak }, 2},
\end{aligned}
$$

where $\quad R_{\text {Auger }, i}=C\left[\left(N_{i}+N_{A i}\right)^{2} N_{i}+\left(N_{i}+N_{A i}\right) N_{i}^{2}\right]$ (Ref. 7) is the Auger nonradiative recombination rate, $J_{i}$ is the carrier recombination current in the $i$ th active layer, $B$ is the radiative recombination constant, $N_{B i}$ is the acceptor dersity in the $i$ th active layer, $A$ is the gain constant, $N_{\text {tr }}$ is the transparency carrier density, $d_{i}$ is the active layer thickness, $\Gamma_{i}$ is the optical mode confinement factor of the $i$ th active layer, $\tau_{p}$ is the photon lifetime, $\beta$ is the coupling constant for spontaneous emission into the lasing mode, and $J_{\text {Leak }, i}$ is the leakage current over the heterobarrier from ith active layer for which the model in Ref. 7 has been adopted.

Using reasonable parameter values, we solved Eqs. (1)(4) numerically. We found that up to threshold the second active layer is absorbing. At threshold the photon density $P$ increases. Also the carrier densities in the two active layers redistribute; a step increase in $N_{2}$ and a step decrease in $N_{1}$ occur. The second active layer is bleached almost to transparency. This accounts for the optical switching behavior of the $\mathrm{BC} D C C$ laser. It is interesting to notice that the feature of the $L-I$ curves depends on the spontaneous emission factor $\beta$. For a real index-guided laser as a $B C$ laser, $\beta$ is small $\left(\sim 10^{-4}\right)$. A jump in output power at threshold is present. However, for gain-guided structures as those in Refs. 4 and $5, \beta$ is much larger, e.g., $\left(5 \times 10^{-3}\right)$, which smears out any optical power jump as confirmed by our calculations. It was also found that the very large $T_{0}$ value cannot be attributed oniy to the carrier leakage. The Auger nonradiative recombination process in this double active layer configuration plays an important role. A detailed description of the calculated results will be published elsewhere.

In conclusion, a BC DCC laser was fabricated by a twostep LPE growth and a "threc-melt technique." The laser showed high characteristic temperature $T_{0}$. Threshold current was typically $2-3$ times higher than that of the single active layer device. An optical switching behavior was observed which may be of practical interest. A theoretical model using the rate equation approach showed good agreement with experimental resuits.

This work was supported by Defence Advanced Research Projects Agency ( $\mathbb{J}$. Murphy) and the Ofice of Naval Research (L. Cooper). 
${ }^{1}$ 1. Mito, M. Kitanura, K. Kobayashi, K. Kobayashi, and R. Lang, presented at the 8th IEEE Int. Semicond. Iaser Conference, Ottawa, Canada, Sept. 13-15, 1982 , paper 28 .

${ }^{2}$ T. R. Chen, L. C. Chiu, K. I. Yu, U. Koren, A. Masson, S. Margahi, and A. Yariv, Jpn. J. Appl. Phys. 22, L559 (1983).

'E. A. Rezek, N. Holonyak, and B. K. Fuller, J. Appl. Phys. 51, 2402 (1980).

${ }^{+}$M. Yano, Y. Nishiani, K-I. Hori, and M. 'akusagawa, IEEE J. Quantum
Electron. QE-19, 1319 (1983).

${ }^{5}$ T. C. Hasenberg and E. M. Garmire, LLEE J. Quantum Llectron. QR-23, $948(1987)$

'E. Oomura, H. Higuchi, Y. Sakakiba, R. Hirano, H. Namizaki, W. Susaki, K. Ikeda, and K. Fujikawa, IEEE J. Quantum Electron. QE-20, 866 (1984).

${ }^{7}$ L. C. Chiv, K. E. Yu, S. Margalit, T. R. Chen, U. Koren, A. Hasson, and A. Yariv, BEEE J. Quantum Electron. QE-19, 1335 (1983) 